\title{
Change in perceived stress and 2-year change in cognitive function among older adults: The Irish Longitudinal Study on Ageing
}

\author{
J. Feeney ${ }^{1,3 *} \mid$ M. O'Sullivan ${ }^{2 *}$ (1) | R.A. Kenny ${ }^{3} \mid$ I.H. Robertson ${ }^{2}$
}

\author{
${ }^{1}$ Centre for Public Health, Queen's University \\ Belfast, Belfast, UK \\ ${ }^{2}$ Neuro-Enhancement for Independent Lives \\ (NEIL), Institute of Neuroscience, Trinity \\ College Dublin, Dublin 2, Ireland \\ ${ }^{3}$ The Irish Longitudinal Study on Ageing, \\ Trinity College Dublin, Dublin 2, Ireland \\ Correspondence \\ Michael O'Sullivan, Neuro-Enhancement for \\ Independent Lives (NEIL), Trinity College \\ Institute of Neuroscience, Trinity College \\ Dublin, Dublin 2, Ireland. \\ Email: michaeo@tcd.ie \\ Funding information \\ The Alzheimer Society of Ireland; The Irish \\ Research Council; the Ageing Research and \\ Development Division of the Institute of Pub- \\ lic Health in Ireland (IPH) formally the Centre \\ for Ageing Research and Development in Ire- \\ land (CARDI); Irish Life plc; The Irish Govern- \\ ment; The Atlantic Philanthropies
}

\begin{abstract}
Prolonged or severe stress can adversely affect older adults' cognitive function, but populationbased studies investigating this relationship over time are rare. Previous studies have largely focused on stress either evaluated at a single time point or linked to specific life events. This study aimed to investigate whether a change in perceived stress over 2 years predicts a change in cognitive performance over the same time period in a population-based sample of older adults. Data from the first 2 waves of The Irish Longitudinal Study on Ageing were analyzed. Five thousand and seventy adults aged 50 and older completed the 4-item Perceived Stress Scale and measures of verbal fluency, immediate and delayed word recall 2 years apart. A first differences regression model revealed that the change in stress over 2 years was inversely associated with a change in immediate word recall performance, even after adjustment for change in possible confounders ( $B=-0.030,95 \% \mathrm{Cl}[-.056,-.004], p<.05)$. No association was observed for delayed recall or verbal fluency performance. Change in perceived stress is inversely correlated with change in immediate recall, even over a short period. Stress modifying interventions could potentially reduce the risk of cognitive decline associated with ageing.
\end{abstract}

\section{KEYWORDS}

ageing, cognition, longitudinal, stress

\section{1 | INTRODUCTION}

One roadblock to understanding the impact of stress exposure on physical health or cognition is the heterogeneity of the individual response to a potential stressor. The appraisal or perception of a situation or event as stressful is important in driving a biological response (Lazarus \& Folkman, 1984), which can occur even in the absence of a stressor (Engert et al., 2013). A number of prominent theories of stress and cognitive decline (Conrad, 2008; Sapolsky, Krey, \& McEwen, 1986) highlight the importance of understanding the role of stress and thus stress perception in ageing.

Numerous studies using animal models have demonstrated the deleterious effect of high or sustained stress exposure on brain architecture, most notably in the hippocampus, amygdala, and prefrontal cortex (see McEwen, Nasca, \& Gray, 2016 for a recent review). In humans, the negative impact of exposure to chronic, severe stress on

*Joint first authors. brain development and cognitive function in children, adolescents, and young adults has also been well documented (De Bellis, Hooper, Spratt, \& Woolley, 2009; Navalta, Polcari, Webster, Boghossian, \& Teicher, 2006; Teicher et al., 2003). Furthermore, in healthy adults, acute stress has been associated with poorer declarative memory (de Quervain Dominique, Roozendaal, Nitsch, McGaugh, \& Hock, 2000; Kuhlmann, Piel, \& Wolf, 2005; Lupien, Gillin, \& Hauger, 1999) and working memory (Young, Sahakian, Robbins, \& Cowen, 1999).

The impact of ongoing (i.e., not incidental) stress on cognition is believed to be potentially greater in older adults where biological defences are less effective (Conrad, 2008; Sapolsky et al., 1986). There is some evidence that chronic stress contributes to late-life cognitive decline (e.g., Johansson et al., 2010; Young et al., 1999). Furthermore, research using older adult caregiving as a model of chronic stress has associated the stress experienced by dementia caregivers with poorer memory, executive function, language skills, and global cognition (de Vugt et al., 2006; Lee, Kawachi, \& Grodstein, 2004), whereas those in equally high stress roles, caring for palliative patients, experienced 
deficits in attention and allocation of attention (Mackenzie, Smith, Hasher, Leach, \& Behl, 2007). The stress hormone cortisol has shown covariations with cognitive function over time among ageing cohorts, particularly with memory (Lee et al., 2007; Li et al., 2006; Lupien et al., 1994; Lupien et al., 1997; Seeman, McEwen, Singer, Albert, \& Rowe, 1997), but also attention and executive function ( $\mathrm{Li}$ et al., 2006; Lupien et al., 1994; Seeman et al., 1997). Higher cortisol levels have also been associated with increased risk of mild cognitive impairment (Peavy et al., 2009).

The contribution of stress perception to age-related decline has been investigated in few studies and often limited to a single measure of stress, but still, they highlight its unique contribution to the trajectory of cognitive performance. Indeed, in older adults, the retrospective reporting of chronic stress appears to be a better predictor of global cognitive decline than the occurrence of life events (Leng et al., 2013; VonDras, Powless, Olson, Wheeler, \& Snudden, 2005). Higher baseline perceived stress has also been found to be associated with poorer cognition and a greater rate of decline in a population sample of older adults over 65 (Aggarwal et al., 2014). Aggarwal and colleagues were able to adjust for multiple covariates measured at a single time point; however, it is unclear whether changes in these covariates could explain the association between baseline perceived stress and subsequent decline. A recent study examined perceived stress and cognition, both assessed at multiple time points over 2 years (Munoz, Sliwinski, Scott, \& Hofer, 2015). They found an association between the level of stress over time and cognitive slowing but no association between the change in stress over time and cognition. Furthermore, the study focused on measures of reaction time in a small group of oldest-old adults, so the association of change in stress with change in memory was not examined. The authors themselves also point out that the advanced age of their sample (mean age of 80 ) likely facilitated the detection of marked cognitive slowing. The question remains as to whether an effect of change in perceived stress on change in performance in other facets of cognition such as memory and semantic fluency (both skills known to be sensitive to early impairment in Alzheimer's disease) exists, particularly in a younger sample in which significant cognitive decline is not yet likely to have occurred, and the impact of any possible interventions to slow decline could be maximized.

The aim of this study was to investigate whether a change in perceived stress over 2 years is associated with concomitant change in cognitive function in a population-based sample of older adults. We hypothesized that an increase in perceived stress over 2 years would be associated with a decline in our three measures of cognition over the same period.

\section{2 | METHODS}

\section{1 | Study design}

The Irish Longitudinal Study on Ageing is a nationally representative prospective cohort study of over 8,000 community dwelling older adults aged 50 years and over. The sampling frame is all residential addresses in the Republic of Ireland (supplied by the Irish
Geodirectory). From this, a clustered sample of addresses were selected, and household residents aged 50 or older and their partners were eligible to participate.

The study design has been described in detail elsewhere (Whelan \& Savva, 2013). Briefly, the data collected comprised (a) a computerassisted personal interview carried out in the participant's own home; (b) a self-completion questionnaire (SCQ); and (c) a health assessment carried out by trained nurses in a dedicated centre or in the respondent's home. Wave 1 took place between 2009 and 2011, and Wave 2 was carried out in 2012-2013; 8,175 individuals aged 50 and older took part in Wave 1, and 7,282 participated in Wave 2. At Wave 2, 80 individuals were no longer cognitively or physically capable of being interviewed in person. A second measure of perceived stress was therefore not recorded for these individuals, and they were necessarily excluded from the analysis.

Ethical approval for the study was obtained from the Trinity College Dublin Ethics committee, and all participants provided written informed consent. This analysis is based on data from the first two waves of the study.

\subsection{Exclusion criteria}

Any individuals who self-reported a doctor's diagnosis of stroke, Parkinson's disease, any dementia or severe memory impairment at Wave 1 were excluded from the analysis. A significant proportion ( $n=1,213$ ) of the Wave 1 sample did not return the SCQ and thus were necessarily excluded. Furthermore, 745 individuals died or dropped out of the study between Waves 1 and 2. Six hundred and three individuals participated in Wave 2 but did not return the Wave 2 SCQ, leaving a sample of 5,398. A further 328 of these were missing data on one or more variables of interest, leaving 5,070 for analysis. Figure 1 details the number of individuals excluded a priori, the number with missing data and the final observed sample for analysis.

\section{3 | Measures}

Stress over the previous month was recorded using the 4-item version of the Perceived Stress Scale (PSS-4; Cohen, Kamarck, \& Mermelstein, 1983) as part of the SCQ. The PSS was originally developed as a 14-item questionnaire, which reflects how unpredictable, uncontrollable, and overloaded a respondent's life is, and is a measure of chronic stress associated with generalized stress perception. The PSS-4 includes four questions answered on a 5-point Likert scale from 0 (Never) to 4 (Very often). A stress change score was created by subtracting Wave 1 from Wave 2 PSS scores.

Cognitive function was assessed with three tasks. Participants underwent a verbal (category) fluency test whereby the participants were asked to name as many animals as possible in $1 \mathrm{~min}$. The score was the total number of animal names generated with the allotted time. Immediate and delayed verbal memory was also assessed using a 10-item word list learning and recall task previously developed for the Health and Retirement Study (Ofstedal, Fisher, \& Herzog, 2005). The 10-item list was read out to and then recalled by participants twice in succession. The total sum of correct items recalled was used as the measure of immediate recall (the maximum score being 20). 


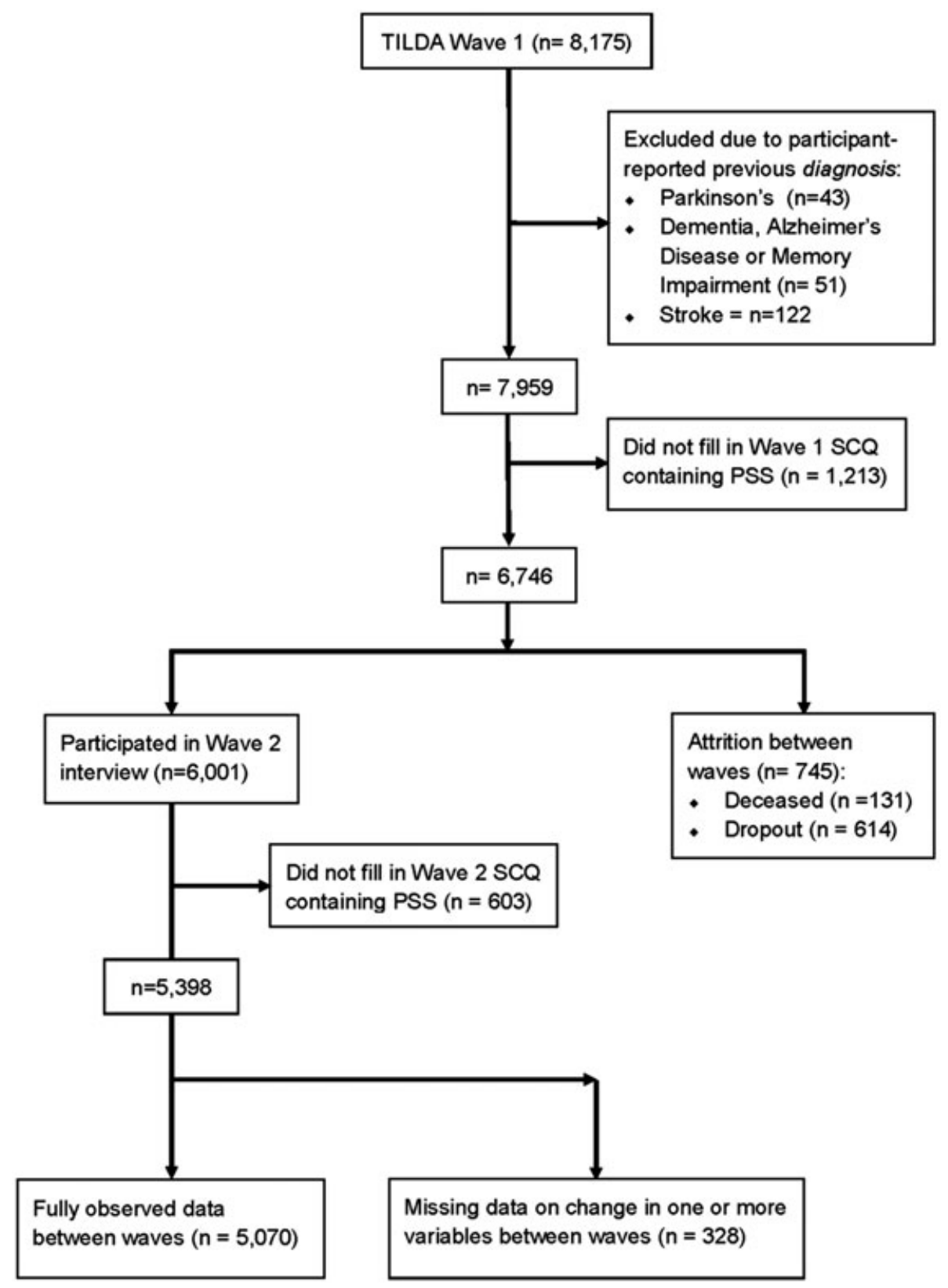

FIGURE 1 Flow diagram of study inclusion. TILDA = Irish Longitudinal Study on Ageing; PSS = Perceived Stress Scale; SCQ = self-completion questionnaire

Approximately 20 min later, participants were asked to recall the list again once only, and the number of correct items recalled comprised the delayed recall score (scored out of 10). Change scores for each cognitive variable were created by subtracting the Wave 1 from Wave 2 score.

All participants' medications were recorded, and a variable was generated to denote the use of any cardiovascular medication. A similar, separate variable was constructed for the use of psychoactive medications. Medications were classified according to the Anatomical Therapeutic Classification (http://www.whocc.no/atc_ddd_index/). Cardiovascular medications were defined as any of the following classes of drugs (Anatomical Therapeutic Classification code in brackets): cardiac therapy ("C01"), antihypertensives ("C02"), diuretics ("C03"), peripheral vasodilators ("C04"), vasoprotectives ("C05"), beta blockers ("C07"), calcium channel blockers ("C08"), ACE inhibitors ("C09"), and lipid modifying agents ("C10"). Psychoactive medications were defined as nervous system drugs comprising anti-Parkinson drugs ("N04"), psycholeptics ("N05") and psychoanaleptics ("N06"), and parasympathomimetics ("N07A"). Change in cardiovascular or psychoactive medication use between waves was noted. Individuals were assigned a 0 if they did not change their medication status between waves, 1 if they started a medication, and -1 if they stopped using any psychoactive or cardiovascular medication.

In order to assess chronic health conditions that might influence both perceived stress and cognitive function, participants were asked at both waves if they had ever been told by a doctor that they had any of the following conditions: angina, heart attack, heart failure, diabetes, ministroke or transient ischaemic attack, high cholesterol, abnormal heart rhythm, heart murmur, asthma, lung disease, arthritis, osteoporosis, cancer, liver disease, cataracts, glaucoma, and age- 
related macular degeneration. A variable was then created representing any increase in the number of self-reported chronic conditions between waves.

Smoking habits were recorded at both waves, and individuals were categorized as having never smoked, being a former smoker, or being a current smoker. Change in smoking status between waves was noted. Individuals were assigned a 0 if they did not change their smoking habits between waves, 1 if they started a smoking, and -1 if they stopped smoking.

At Wave 2, the Composite International Diagnostic Interview Short Form (Kessler, Andrews, Mroczek, Ustun, \& Wittchen, 1998) for major depression was included as part of the computer-assisted personal interview. This instrument is designed to identify episodes of major depression during the previous year. A diagnostic algorithm is employed to determine whether an individual meets the criteria for a major depressive episode or not. This yielded a dichotomous variable for inclusion in further analysis, whereby individuals meeting the criteria for a major depressive episode were assigned a "1," and those who did not meet the criteria, a "0."

\section{4 | Statistical analyses}

The distribution of all variables of interest was initially examined via histograms and Q-Q plots. Only cases with data on all variables of interest were included in this analysis. Seventy-seven responders with missing data on a single item from the PSS-4 only were included in the change score analysis by estimating (prorating) the missing item score from an average of the completed items. The $t$ test and chi-squared test were used to compare individuals included and excluded from the analysis on continuous outcomes (e.g., perceived stress and cognition) and categorical covariates (e.g., sex, education, and smoking), respectively (Table 1). Bivariate tests of association between Waves
1 and 2 cognitive scores were carried out using $t$ tests, and differences in perceived stress between Waves 1 and 2 were investigated using the sign test (results in Table 2).

Our multivariable analysis incorporates the "first difference" analytic approach, which underpins common repeated measures statistical tests such as $t$ tests and analysis of variance (Thomas \& Zumbo, 2012) and is equivalent to a fixed-effects panel data model when two waves of data are used. Presenting results in the form of the difference approach aids interpretability as difference scores are often considered a natural measure of change (Gottman \& Rushe, 1993). The reliability of difference scores has been found to be high in tests with only moderate test-retest reliability (Rogosa, 1995). The repeated measures nature of the fixed effects model means each person is, in effect, their own control. Thus, time-invariant characteristics should not be adjusted for (i.e., age of entry into the study, gender, previous educational attainment, and any other demographic characteristics that would not be expected to change differently for different individuals between waves). Similarly, it is often argued that adjustment for baseline is necessary when analyzing change. This would not only make our model less interpretable; the introduction of a baseline a covariate commonly introduces bias into regression models (Glymour, Weuve, Berkman, Kawachi, \& Robins, 2005).

TABLE 2 Change in PSS and cognition between waves

\begin{tabular}{lrrr} 
& \multicolumn{1}{c}{ Wave 1 } & \multicolumn{1}{c}{ Wave 2 } & \multicolumn{1}{c}{$\boldsymbol{p}$} \\
\cline { 2 - 4 } Variable & \multicolumn{1}{c}{ Mean (SD) } & & $* * *$ \\
\hline Immediate recall & $13.66(3.03)$ & $13.91(3.05)$ & - \\
Delayed recall & $6.20(2.23)$ & $6.17(2.48)$ & - \\
Category fluency & $21.36(6.85)$ & $19.70(6.02)$ & $* * *$ \\
Stress & $4.05(3.08)$ & $4.35(3.00)$ & $* * *$ \\
\hline
\end{tabular}

Note. PSS = Perceived Stress Scale; SD = standard deviation . ${ }^{* * *} p<.001 ;-p>.05$.

TABLE 1 A comparison of the baseline characteristics of the individuals in the analytic sample versus those individuals with missing data

\begin{tabular}{|c|c|c|}
\hline \multirow[b]{2}{*}{ Variable } & Analytic sample & Individuals with missing data \\
\hline & \multicolumn{2}{|l|}{$\% /$ Mean $(S D)$, range } \\
\hline Age & 62.86 (9.01), 50-93 & 65.05 (10.73), 50-105*** \\
\hline Education to tertiary level & 34.25 & $21.55^{* * *}$ \\
\hline \multicolumn{3}{|l|}{ Wave 1} \\
\hline Delayed recall & 6.20 (2.23), $1-10$ & 5.35 (2.42), $1-20^{* * *}$ \\
\hline PSS score & 4.05 (3.07), 0-16 & $4.86(3.20), 0-16^{* * *}$ \\
\hline Cardiovascular medication use & 48.86 & $49.88^{\text {ns }}$ \\
\hline Psychotropic medication use & 9.11 & $12.56^{* * *}$ \\
\hline Smoking & 15.15 & $23.61^{* * *}$ \\
\hline
\end{tabular}

Note. $\mathrm{ns}=$ nonsignificant; $S D=$ standard deviation.

${ }^{* * *} p<.001$. 
TABLE 3 Linear regression analysis of change in cognition on change in stress between waves

\begin{tabular}{|c|c|c|c|c|c|c|}
\hline & \multicolumn{2}{|c|}{ Total immediate recall } & \multicolumn{2}{|c|}{ Delayed recall } & \multicolumn{2}{|c|}{ Category fluency } \\
\hline & B & $95 \% \mathrm{Cl}$ & B & $95 \% \mathrm{Cl}$ & B & $95 \% \mathrm{Cl}$ \\
\hline Model 1 & $-0.030^{*}$ & {$[-.056,-.004]$} & 0.002 & {$[-.018, .021]$} & -0.026 & {$[-.083, .031]$} \\
\hline Model 3 & $-0.030^{*}$ & {$[-.056,-.004]$} & 0.002 & {$[-.018, .021]$} & -0.026 & {$[-.083, .031]$} \\
\hline
\end{tabular}

Note. Model 1 unadjusted; Model 2 adjusted for change in psychotropic and cardiovascular medication use and for the increase in chronic conditions between waves. Model 3 further adjusted for change in smoking and major depressive episode between waves.

${ }^{*} p<.05$.

Three linear regression models were used to interrogate the relationship between change in stress and change in cognition. Model 1 describes the bivariate relationship between change in stress and change in cognitive performance. Subsequent models were run with adjustment for potential time-varying covariates. Model 2 was adjusted for change in medication status and change in the number of chronic conditions. Model 3 was further adjusted for change in smoking habits and experience of a major depressive episode in the previous 12 months. Standard errors were corrected for the stratified-clustered survey design. Data were analyzed using Stata Version 12 (StataCorp, College Station, TX, USA).

\section{3 | RESULTS}

\section{1 | Baseline characteristics of individuals in the analytic sample and those excluded from analysis}

Five thousand seventy participants completed the recall and verbal fluency tests and the perceived stress scale at both waves as well as questions relating to chronic conditions, smoking, and the use of cardiovascular and psychotropic medications (see Figure 1). In comparison with those necessarily excluded from the study ( $n=3,105)$, those included in the analysis were younger, more educated, less likely to be taking psychotropic medications, and less stressed. They also performed significantly better on each of the three measures of cognition than those who were excluded. There was no difference between the groups in sex, frequency of chronic conditions, or cardiovascular medication use (Table 1).

\subsection{Change in cognition, perceived stress, and covariates between waves in the analytic sample}

The mean change in cognitive and perceived stress scores between waves for the sample as a whole is displayed in Table 2. Mean verbal fluency score declined over the 2 years. A slight mean increase in performance was observed on the immediate recall task; however, no change in performance on the delayed recall task between waves was evident. There was a mean increase in PSS scores between waves.

There was considerable change in medication use between Waves 1 and 2. 123 participants who were taking any psychotropic medication at Wave 1 were no longer doing so at Wave 2, whereas 278 participants who were not taking psychotropics at Wave 1 starting taking a medication between waves. 182 participants stopped taking any cardiovascular medication between waves. By contrast, 606 participants started on a cardiovascular medication between waves.
The number of doctor diagnosed chronic health conditions reported by the sample also increased between waves. 968 individuals reported one new chronic condition at Wave 2; whereas 146 reported an increase of two or more conditions between waves. There was minimal change in smoking habits between waves; 137 individuals stopped smoking, whereas only 39 individuals reporting taking up the habit. At Wave 2, 280 individuals met the criteria for having experienced major depressive episode in the previous year.

\section{3 | Multiple linear regression analysis of the change in cognition associated with the change in the level of perceived stress between Waves 1 and 2}

The association between the change in cognitive scores and change in stress is displayed in Table 3. An increase in perceived stress was significantly associated with a change in immediate recall between waves in the unadjusted model ( $\beta=-0.030,95 \% \mathrm{Cl}[-.056,-.004], p=.022$ ). The association remained after adjustment for change in medication use and new chronic conditions (Model 2). Further adjustment for change in smoking and occurrence of a major depressive episode between waves did not attenuate this association ${ }^{1}$. There was no evidence for an association between change in stress and change in delayed recall or verbal fluency score between waves. Figure 2 shows the standardized change in each of the cognitive variables for a standard deviation increase in PSS score between waves.

\section{4 | DISCUSSION}

Over 2 years, change in immediate word recall was found to be negatively associated with a change in perceived stress in this large ageing population-based cohort study. The association survived correction for change in a number of behavioural/health variables, derived from variables measured at both time points, including smoking status, psychotropic and cardiovascular medication use, incidence of chronic conditions, and incidence of depression over 2 years. No association was found between change in perceived stress and semantic fluency or delayed recall over 2 years in our sample. Overall, our results support the interpretation that increased stress is associated with

${ }^{1}$ In response to reviewer comments, a sensitivity analysis adding age, gender, and educational attainment to our models was completed. This did not alter the size of the coefficients appreciably. This was anticipated as these are timeinvariant characteristics. An increase in perceived stress over the 2 -year period remained associated with the change in immediate recall performance over the same period at the $5 \%$ level. 


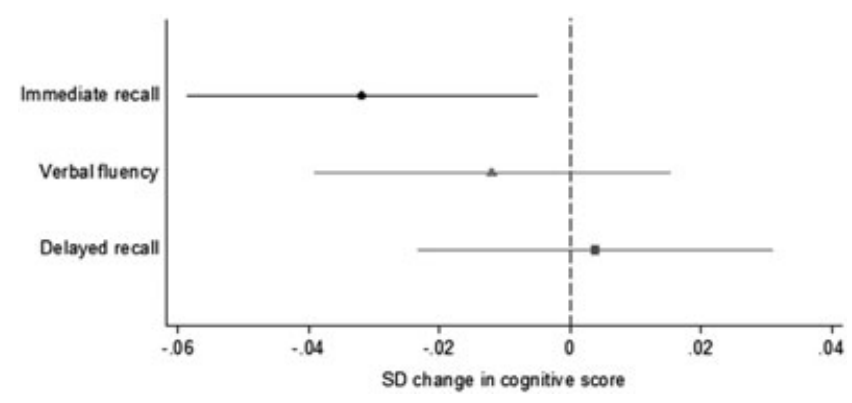

FIGURE 2 Standardized change in cognition per standard deviation (SD) increase in Perceived Stress Scale score over 2 years

decreased immediate recall performance. Our results extend those of Munoz et al. (2015) by examining other domains of cognition and investigating change in performance accuracy rather than reaction time, in a large sample with a wide age range.

To the authors' knowledge, no other study to date has demonstrated an association between a change in perceived stress as measured by the PSS and change in cognition among older adults. Previous work has shown that perceived stress measured at baseline affects the rate of change of cognition over 7 years (Aggarwal et al., 2014) and that estimates of previous stress are associated with poorer cognition (Leng et al., 2013) or incident dementia (Johansson et al., 2010). A recent study showed that over 2 -year cognitive slowing was related to the overall level of stress reported at six time points (Munoz et al., 2015). Studies focusing on life events have demonstrated that experiencing certain life events (e.g., death of a child and financial trouble) is associated with poorer cognition (Comijs, van den Kommer, Minnaar, Penninx, \& Deeg, 2011; Rosnick, Small, McEvoy, Borenstein, \& Mortimer, 2007). However, the latter studies also showed that the occurrence of some stressors of a more chronic nature (e.g., the illness of a friend or relative) and the perception by the individual that the event had a greater impact on their life was associated with better cognition. These divergent findings with respect to event type and the strength of the perceived impact of the event highlight the importance of the subjective appraisal of stress in predicting health outcomes, which the results of the current study also support.

One possible explanation of the association between perceived stress and immediate word recall observed here is that increasing levels of stress-related cortisol have a deleterious effect on areas of the brain including the prefrontal cortex and the hippocampus in older adults; both of which contain high levels of the glucocorticoid receptor (Ulrich-Lai \& Herman, 2009), and both of which are implicated in memory acquisition and recall processes (Blumenfeld \& Ranganath, 2007; Eldridge, Engel, Zeineh, Bookheimer, \& Knowlton, 2005; Nyberg et al., 2003; Ranganath \& D'Esposito, 2001). The high density of glucocorticoid receptors in these regions may confer vulnerability to cortisol's deleterious effects (Sapolsky, 1986) with higher cortisol levels associated with a greater risk of neuronal death (Conrad, 2008). An alternative explanation may be that individuals who have experienced an increase in stress may have fewer attentional resources available to allocate to a task at hand, which may manifest as more task irrelevant thoughts and poorer performance under greater cognitive load (e.g., Eysenck, Derakshan, Santos, \& Calvo,
2007; Kahnemann, 1973; Sarason, Sarason, Keefe, Hayes, \& Shearin, 1986). It is noteworthy that we did not observe an effect of stress on the delayed recall task in addition to immediate recall. We can but speculate as to why this was the case. The initial acquisition and consolidation of verbal material taxes attentional processes that could arguably have a greater impact on the immediate recall of the material than on the delayed recall task. This would again support the hypothesis regarding allocation or availability of attentional resources, rather than a true effect on long-term memory. In addition, the latter task has no learning component and is by nature more susceptible to forgetting, reducing the variability in scores, and thus power to detect an effect. This contrasts with the immediate recall task where individuals got two chances to recall the items, and their score for each was summed to give a possible maximum score of 20 (versus 10 for the delayed recall task).

Over the whole sample, perceived stress increased between waves. There is evidence that an overall trend for decreasing levels of perceived stress over long time scales typically occurs in older adults as stress perception is an inherently adaptive process (Vasunilashorn, Lynch, Glei, Weinstein, \& Goldman, 2014). However, our data were collected over 2 years, and owing to this shorter timescale, the increase may be related to significant life events occurring between waves or financial and associated stress that increased at the population level against a backdrop of harsh economic recession and consequent austerity through which the Irish people suffered during this period. Interestingly, Hauksdóttir, McClure, Hrafn Jonsson, Ólafsson, and Valdimarsdóttir (2013) reported similar mean perceived stress levels and a similar increase in stress levels in population sample of older adults in Iceland, spanning the most recent period of economic collapse in the country. The average perceived stress levels reported in our sample are similar to or even slightly higher than the mean scores previously reported in community-dwelling older adult cohorts using the PSS-4 (e.g., Cohen \& Williamson, 1988; Hauksdóttir et al., 2013; McClellan et al., 2010; Wiernik et al., 2016). There was also a large range of PSS change scores evident, with a handful of individuals experiencing very large increases or decreases in stress between waves ( \pm 3 standard deviation from the mean). However, removing these individuals from the analysis did not attenuate the effect at all, indicating that the association between stress and immediate recall is not unduly influenced by these more extreme change scores.

The strengths of this study include the large population-based sample used and the assessment of both perceived stress and cognition at two time points. This type of model negates the requirement to adjust for known time invariant individual characteristics and also lessens the possibility of confounding from unobserved variables. A further strength of our study is the adjustment for change in behavioural and health covariates that could potentially explain the association between stress and cognition over time. Limitations of this study include that we have only two waves of data with approximately 2 years between baseline and follow-up, which is a short time within which to expect cognitive decline to occur in a relatively healthy and young cohort. A significant practice effect was evident in the immediate recall scores for the sample as a whole, thus caution should be exercised in interpreting the results; however, they are in line with the findings reported by other related studies of stress and cognition 
(e.g., Comijs et al., 2011), as well as with reports of longitudinal cognitive trajectories from other cohort studies (e.g., Tombaugh, 2005). Another limitation is that the longitudinal study sample with complete data on variables of interest comprised participants who were younger, more educated, had better baseline cognition, and were less stressed than those with missing data, which should be taken into consideration when interpreting the findings. Although we were able to adjust for change in several potential covariates between waves, a high level of longitudinal missingness on other variables such as problem alcohol use meant that we could not explore the impact of change in this variable on the stress-cognition relationship. There will be some regression attenuation due to measurement error in the PSS scale, leading to conservative estimates. We cannot be certain as to the direction of causality based on two waves of data. It is possible that awareness of declining cognition over time led individuals to report increased stress at Wave 2. However, we carried out a sensitivity analysis further adjusting for self-reported memory change between waves and found that this did not change the results, so we believe that reverse causality is unlikely to be at play here.

Although our results are consistent with those of other groups showing that stress perception is an important factor in cognitive decline (e.g., Aggarwal et al., 2014; Johansson et al., 2010; Munoz et al., 2015), it would be useful in future to measure objective indices of stress such as cortisol levels in tandem with subjective reporting, to better investigate the biological and psychological mechanisms through which chronic stress among older adults negatively affects the brain.

In conclusion, we found that an increase in perceived stress over 2 years was inversely associated with a change in immediate word recall performance over the same time period in a population study of adults aged 50 years and older. This finding suggests a potential role for stress reduction techniques, for example, increased physical activity and social engagement, mindfulness, or even cognitive behaviour therapy for chronically stressed individuals in midlife and young older age, to reduce the risk of long-term cognitive decline.

\section{FUNDING}

This work was supported by The Atlantic Philanthropies, The Irish Government, Irish Life plc, the Ageing Research and Development Division of the Institute of Public Health in Ireland (IPH) formally the Centre for Ageing Research and Development in Ireland (CARDI), The Irish Research Council, and The Alzheimer Society of Ireland.

\section{ACKNOWLEDGMENTS}

The authors would like to thank all the TILDA participants and Dr. Neil O'Leary for his statistical advice.

\section{ORCID}

M. O'Sullivan 19 http://orcid.org/0000-0003-0055-599X

\section{REFERENCES}

Aggarwal, N. T., Wilson, R. S., Beck, T. L., Rajan, K. B., Mendes de Leon, C. F., Evans, D. A., \& Everson-Rose, S. A. (2014). Perceived stress and change in cognitive function among adults 65 years and older. Psychosomatic Medicine, 76(1), 80-85. https://doi.org/10.1097/psy. 0000000000000016
Blumenfeld, R. S., \& Ranganath, C. (2007). Prefrontal cortex and long-term memory encoding: An integrative review of findings from neuropsychology and neuroimaging. The Neuroscientist, 13(3), 280-291. https://doi.org/ $10.1177 / 1073858407299290$

Cohen, S., Kamarck, T., \& Mermelstein, R. (1983). A global measure of perceived stress. Journal of Health and Social Behavior, 385-396.

Cohen, S., \& Williamson, G. M. (1988). Perceived stress in a probability sample of the United States. In S. Spacapan, \& S. Oskamp (Eds.), The social psychology of health. Newbury Park, CA: Sage.

Comijs, H. C., van den Kommer, T. N., Minnaar, R. W., Penninx, B. W., \& Deeg, D. J. (2011). Accumulated and differential effects of life events on cognitive decline in older persons: Depending on depression, baseline cognition, or ApoE epsilon4 status? The Journals of Gerontology. Series B, Psychological Sciences and Social Sciences, 66(Suppl 1), i111-i120. https://doi.org/10.1093/geronb/gbr019

Conrad, C. D. (2008). Chronic stress-induced hippocampal vulnerability: The glucocorticoid vulnerability hypothesis. Reviews in the Neurosciences, 19(6), 395-412.

De Bellis, M. D., Hooper, S. R., Spratt, E. G., \& Woolley, D. P. (2009). Neuropsychological findings in childhood neglect and their relationships to pediatric PTSD. Journal of the International Neuropsychological Society, 15(6), 868-878. https://doi.org/10.1017/s1355617709990464

de Quervain Dominique, J.-F., Roozendaal, B., Nitsch, R. M., McGaugh, J. L., $\&$ Hock, C. (2000). Acute cortisone administration impairs retrieval of long-term declarative memory in humans. Nature Neuroscience, 3(4), 313-314.

de Vugt, M. E., Jolles, J., van Osch, L., Stevens, F., Aalten, P., Lousberg, R., \& Verhey, F. R. (2006). Cognitive functioning in spousal caregivers of dementia patients: Findings from the prospective MAASBED study. Age and Ageing, 35(2), 160-166. https://doi.org/10.1093/ageing/ afj044

Eldridge, L. L., Engel, S. A., Zeineh, M. M., Bookheimer, S. Y., \& Knowlton, B. J. (2005). A dissociation of encoding and retrieval processes in the human hippocampus. The Journal of Neuroscience, 25(13), 3280-3286. https://doi.org/10.1523/jneurosci.3420-04.2005

Engert, V., Efanov, S. I., Duchesne, A., Vogel, S., Corbo, V., \& Pruessner, J. C. (2013). Differentiating anticipatory from reactive cortisol responses to psychosocial stress. Psychoneuroendocrinology, 38(8), 1328-1337. https://doi.org/10.1016/j.psyneuen.2012.11.018

Eysenck, M. W., Derakshan, N., Santos, R., \& Calvo, M. G. (2007). Anxiety and cognitive performance: Attentional control theory. Emotion, 7(2), 336.

Glymour, M. M., Weuve, J., Berkman, L. F., Kawachi, I., \& Robins, J. M. (2005). When is baseline adjustment useful in analyses of change? An example with education and cognitive change. American Journal of Epidemiology, 162(3), 267-278.

Gottman, J. M., \& Rushe, R. H. (1993). The analysis of change: Issues, fallacies, and new ideas. Journal of Consulting and Clinical Psychology, 61(6), 907.

Hauksdóttir, A., McClure, C., Hrafn Jonsson, S., Ólafsson, O., \& Valdimarsdóttir, U. A. (2013). Increased stress among women following an economic collapse-A prospective cohort study. American Journal of Epidemiology, 177(9), 979-988.

Johansson, L., Guo, X., Waern, M., Östling, S., Gustafson, D., Bengtsson, C., \& Skoog, I. (2010). Midlife psychological stress and risk of dementia: A 35-year longitudinal population study. Brain, 133(8), 2217-2224. https://doi.org/10.1093/brain/awq116

Kahnemann, D. (1973). Attention and Effort.

Kessler, R. C., Andrews, G., Mroczek, D., Ustun, B., \& Wittchen, H. U. (1998). The World Health Organization Composite International Diagnostic Interview Short-Form (CIDI-SF). International Journal of Methods in Psychiatric Research, 7(4), 171-185.

Kuhlmann, S., Piel, M., \& Wolf, O. T. (2005). Impaired memory retrieval after psychosocial stress in healthy young men. The Journal of Neuroscience, 25(11), 2977-2982. https://doi.org/10.1523/JNEUROSCI.5139-04.2005 
Lazarus, R. S., \& Folkman, S. (1984). Stress appraisal and coping.

Lee, B. K., Glass, T. A., McAtee, M. J., Wand, G. S., Bandeen-Roche, K., Bolla, K. I., \& Schwartz, B. S. (2007). Associations of salivary cortisol with cognitive function in the Baltimore memory study. Archives of General Psychiatry, 64(7), 810-818. https://doi.org/10.1001/archpsyc.64.7.810

Lee, S., Kawachi, I., \& Grodstein, F. (2004). Does caregiving stress affect cognitive function in older women? The Journal of Nervous and Mental Disease, 192(1), 51-57.

Leng, Y., Wainwright, N. W., Hayat, S., Stephan, B. C., Matthews, F. E., Luben, R., ... Brayne, C. (2013). The association between social stress and global cognitive function in a population-based study: The European Prospective Investigation into Cancer (EPIC)-Norfolk study. Psychological Medicine, 43(3), 655-666. https://doi.org/10.1017/ s0033291712001316

Li, G., Cherrier, M. M., Tsuang, D. W., Petrie, E. C., Colasurdo, E. A., Craft, S., ... Wilkinson, C. W. (2006). Salivary cortisol and memory function in human aging. Neurobiology of Aging, 27(11), 1705-1714. https://doi. org/10.1016/j.neurobiolaging.2005.09.031

Lupien, S., Gaudreau, S., Tchiteya, B., Maheu, F., Sharma, S., Nair, N., ... Meaney, M. (1997). Stress-induced declarative memory impairment in healthy elderly subjects: Relationship to cortisol reactivity 1 . The Journal of Clinical Endocrinology and Metabolism, 82(7), 2070-2075.

Lupien, S., Lecours, A. R., Lussier, I., Schwartz, G., Nair, N. P., \& Meaney, M. J. (1994). Basal cortisol levels and cognitive deficits in human aging. The Journal of Neuroscience, 14(5 Pt 1), 2893-2903.

Lupien, S. J., Gillin, C. J., \& Hauger, R. L. (1999). Working memory is more sensitive than declarative memory to the acute effects of corticosteroids: A dose-response study in humans. Behavioral Neuroscience, 113(3), 420.

Mackenzie, C. S., Smith, M. C., Hasher, L., Leach, L., \& Behl, P. (2007). Cognitive functioning under stress: Evidence from informal caregivers of palliative patients. Journal of Palliative Medicine, 10(3), 749-758.

McClellan, W. M., Abramson, J., Newsome, B., Temple, E., Wadley, V. G., Audhya, P., ... Kimmel, P. (2010). Physical and psychological burden of chronic kidney disease among older adults. American Journal of Nephrology, 31(4), 309-317.

McEwen, B. S., Nasca, C., \& Gray, J. D. (2016). Stress effects on neuronal structure: Hippocampus, amygdala, and prefrontal cortex. Neuropsychopharmacology, 41(1), 3-23.

Munoz, E., Sliwinski, M. J., Scott, S. B., \& Hofer, S. (2015). Global perceived stress predicts cognitive change among older adults. Psychology and Aging, 30(3), 487-499. https://doi.org/10.1037/pag0000036

Navalta, C. P., Polcari, A., Webster, D. M., Boghossian, A., \& Teicher, M. H. (2006). Effects of childhood sexual abuse on neuropsychological and cognitive function in college women. The Journal of Neuropsychiatry and Clinical Neurosciences, 18(1), 45-53. https://doi.org/10.1176/ jnp.18.1.45

Nyberg, L., Marklund, P., Persson, J., Cabeza, R., Forkstam, C., Petersson, K. M., \& Ingvar, M. (2003). Common prefrontal activations during working memory, episodic memory, and semantic memory. Neuropsychologia, 41(3), 371-377.

Ofstedal, M. B., Fisher, G. G., \& Herzog, A. R. (2005). Documentation of cognitive functioning measures in the Health and Retirement Study. Ann Arbor, MI: University of Michigan.

Peavy, G. M., Salmon, D. P., Jacobson, M. W., Hervey, A., Gamst, A. C., Wolfson, T., ... Galasko, D. (2009). Effects of chronic stress on memory decline in cognitively normal and mildly impaired older adults. The American Journal of Psychiatry, 166(12), 1384-1391. https://doi.org/ 10.1176/appi.ajp.2009.09040461

Ranganath, C., \& D'Esposito, M. (2001). Medial temporal lobe activity associated with active maintenance of novel information. Neuron, 31(5), 865-873.
Rogosa, D. (1995). Myths and methods:"Myths about longitudinal research" plus supplemental questions. The Analysis of Change, 3, 66.

Rosnick, C. B., Small, B. J., McEvoy, C. L., Borenstein, A. R., \& Mortimer, J. A. (2007). Negative life events and cognitive performance in a population of older adults. Journal of Aging and Health, 19(4), 612-629. https://doi.org/ 10.1177/0898264307300975

Sapolsky, R. (1986). Glucocorticoid toxicity in the hippocampus. Neuroendocrinology, 43(3), 440-444.

Sapolsky, R. M., Krey, L. C., \& McEwen, B. S. (1986). The neuroendocrinology of stress and aging: The glucocorticoid cascade hypothesis. Endocrine Reviews, 7(3), 284-301.

Sarason, I. G., Sarason, B. R., Keefe, D. E., Hayes, B. E., \& Shearin, E. N. (1986). Cognitive interference: Situational determinants and traitlike characteristics. Journal of Personality and Social Psychology, 51(1), 215-226. https://doi.org/10.1037/0022-3514.51.1.215

Seeman, T. E., McEwen, B. S., Singer, B. H., Albert, M. S., \& Rowe, J. W. (1997). Increase in urinary cortisol excretion and memory declines: MacArthur studies of successful aging. The Journal of Clinical Endocrinology and Metabolism, 82(8), 2458-2465. https://doi.org/10.1210/ jcem.82.8.4173

Teicher, M. H., Andersen, S. L., Polcari, A., Anderson, C. M., Navalta, C. P., \& Kim, D. M. (2003). The neurobiological consequences of early stress and childhood maltreatment. Neuroscience \& Biobehavioral Reviews, 27(1), 33-44.

Thomas, D. R., \& Zumbo, B. D. (2012). Difference scores from the point of view of reliability and repeated-measures ANOVA in defense of difference scores for data analysis. Educational and Psychological Measurement, 72(1), 37-43.

Tombaugh, T. N. (2005). Test-retest reliable coefficients and 5-year change scores for the MMSE and 3MS. Archives of Clinical Neuropsychology, 20(4), 485-503. https://doi.org/10.1016/j.acn.2004.11.004

Ulrich-Lai, Y. M., \& Herman, J. P. (2009). Neural regulation of endocrine and autonomic stress responses. Nature Reviews. Neuroscience, 10(6), 397-409. https://doi.org/10.1038/nrn2647

Vasunilashorn, S., Lynch, S. M., Glei, D. A., Weinstein, M., \& Goldman, N. (2014). Exposure to stressors and trajectories of perceived stress among older adults. The Journals of Gerontology Series B: Psychological Sciences and Social Sciences.. https://doi.org/10.1093/geronb/gbu065

VonDras, D. D., Powless, M. R., Olson, A. K., Wheeler, D., \& Snudden, A. L. (2005). Differential effects of everyday stress on the episodic memory test performances of young, mid-life, and older adults. Aging \& Mental Health, 9(1), 60-70.

Whelan, B. J., \& Savva, G. M. (2013). Design and methodology of the Irish Longitudinal Study on Ageing. Journal of the American Geriatrics Society, 61(s2), S265-S268.

Wiernik, E., Lemogne, C., Thomas, F., Perier, M. C., Guibout, C., Nabi, H., ... Empana, J. P. (2016). Perceived stress, common carotid intima media thickness and occupational status: The Paris Prospective Study III. International Journal of Cardiology, 15(221), 1025-1030.

Young, A., Sahakian, B., Robbins, T., \& Cowen, P. (1999). The effects of chronic administration of hydrocortisone on cognitive function in normal male volunteers. Psychopharmacology, 145(3), 260-266.

How to cite this article: Feeney J, O'Sullivan M, Kenny RA, Robertson IH. Change in perceived stress and 2-year change in cognitive function among older adults: The Irish Longitudinal Study on Ageing. Stress and Health. 2018;1-8. https://doi.org/ 10.1002/smi.2799 